\title{
Diagnóstico de las necesidades de educación formal en el área de las Tecnologías de Información y Comunicación en Costa Rica, 2016-2019
}

Diagnostics on formal education needs in Information and Communication Technological Areas in Costa Rica, 2016-2019

\section{Volumen 20, Número 2 \\ Mayo - Agosto \\ pp. 1-31}

\author{
Sindy Porras Santamaría \\ Alberto Ramírez Molina \\ Verni Fernández Castro
}

\section{Citar este documento según modelo APA}

Porras Santamaría, Sindy., Ramírez Molina, Alberto. y Fernández Castro, Verni. (2020). Diagnóstico de las necesidades de educación formal en el área de las Tecnologías de Información y Comunicación en Costa Rica, 2016-2019. Revista Actualidades Investigativas en Educación, 20(2), 1-31. Doi. 10.15517/aie.v20i2.41642 


\title{
Diagnóstico de las necesidades de educación formal en el área de las Tecnologías de Información y Comunicación en Costa Rica, 2016-2019
}

Diagnostics on formal education needs in Information and Communication Technological Areas in Costa Rica, 2016-2019

\author{
Sindy Porras Santamaría ${ }^{1}$ \\ Alberto Ramírez Molina ${ }^{2}$ \\ Verni Fernández Castro ${ }^{3}$
}

\begin{abstract}
Resumen: Ante los constantes avances de las Tecnologías de la Información y la Comunicación (TIC) surge la inquietud de conocer si las personas graduadas de las carreras afines a las TIC cuentan con los conocimientos y aptitudes que requiere la sociedad costarricense. Por ello, se plantea la oportunidad de realizar un diagnóstico que considere la teoría existente, la opinión de empresas empleadoras y la oferta académica que ofrecen las universidades públicas con el fin de identificar aspectos en los que se pueda mejorar. En el presente artículo se trabajó bajo una metodología cualitativa, realizando una revisión documental de los estudios recientes a nivel nacional relacionados con las TIC y los planes de estudio de las universidades públicas de Costa Rica: Instituto Tecnológico de Costa Rica (TEC), Universidad de Costa Rica (UCR), Universidad Nacional (UNA), Universidad Estatal a Distancia (UNED) y Universidad Técnica Nacional (UTN). Además de veintiséis representantes de empresas empleadoras de la población graduada en el área, a fin de comparar perspectivas. Se obtuvo como resultado un listado de las necesidades de mejora en la educación formal, entre los que encuentran las habilidades blandas, especialización en temáticas como Big Data, Deep Learning o Internet de las cosas y la alta demanda insatisfecha de profesionales. Por último, se destacan los aportes del estudio como insumo en procesos de actualización de perfil de salida, en la elaboración de nuevas opciones académicas o en la creación de programas de actualización continua.
\end{abstract}

Palabras clave: TIC, educación formal, educación superior

\begin{abstract}
Due to the constant improvements of the Information and Communication Technologies (ICT), a concern came up to figure out if the major graduates on ICT careers have the knowledge and skills required by the Costa Rican society. Therefore, an opportunity was suggested to test the current theory, the employers opinions and the academic offer in public universities which will provide and identify aspects where there might be room for improvement. In the article provided, a qualitative methodology was utilized by doing a documental revision from recent studies at a national level related to ICT and the study curriculum of public universities in Costa Rica: Instituto Tecnológico de Costa Rica (TEC), Universidad de Costa Rica (UCR), Universidad Nacional (UNA), Universidad Estatal a Distancia (UNED) y Universidad Técnica Nacional (UTN). Furthermore, twenty six stakeholders from information technology companies were involved with graduates in this area, which provided a list of needs and improvements in the formal education. Among the aspects found, soft skills, specializations like Big Data, Deep Learning or Internet of things and the high demand of unsatisfied professionals. At last, a highlight of the study contributions input in the current final profile process, in the elaboration of new academic options o in the creation of current continuing programs.
\end{abstract}

Keywords: ICT, formal education, higher education

1 Docente e Investigadora de la Universidad de Costa Rica, Sede de Occidente, Costa Rica. Dirección electrónica: sindy.porras@ucr.ac.cr. Orcid https://orcid.org/0000-0003-0229-1801

2 Docente e Investigador de la Universidad de Costa Rica, Sede de Occidente, Costa Rica. Dirección electrónica: alberto.ramirezmolina@ucr.ac.cr. Orcid https://orcid.org/0000-0001-8711-8594

${ }^{3}$ Docente e Investigador de la Universidad de Costa Rica, Sede de Occidente, Costa Rica. Dirección electrónica: verny.fernandez@ucr.ac.cr. Orcid https://orcid.org/0000-0002-4638-341X

Artículo recibido: 30 de octubre, 2019

Enviado a corrección: 28 de febrero, 2020

Aprobado: 20 de abril, 2020 


\section{Introducción}

Este artículo se centra en dar a conocer el diagnóstico de las necesidades reales del mercado actual de Costa Rica en el área de las Tecnologías de Información y Comunicación, en adelante TIC, tomando como base los planes de estudio de las carreras afines de las universidades públicas, con el fin de identificar las temáticas que deben fortalecerse 0 agregarse a las opciones ya existentes, y en las que se desarrollen en un futuro cercano.

Todas las universidades públicas ofrecen variedad de opciones académicas de pregrado y postgrado en el área de las TIC en diferentes regiones del país, permitiéndole al estudiantado elegir y, en algunos casos, poder cursar una de estas opciones en una sede regional cercana a su lugar de procedencia. Sin embargo, el porcentaje de las personas graduadas a nivel universitario en el área se ha mantenido alrededor del $8 \%$ en los últimos años, lo que representa un aproximado de 3700 personas al año (Programa Sociedad de la Información y el Conocimiento, 2018).

Los avances en las TIC hacen que se desarrolle un constante cambio tecnológico aunado a las capacidades que tienen las empresas para asumirlos (Arévalo-Ascanio, Bayona-Trillos y Rico-Bautista, 2015), invirtiendo en ellos para lograr ventajas competitivas. De la mano con estos avances, se requiere también contar con el personal capacitado, lo que requiere una actualización de las competencias profesionales (Morato, SánchezCuadrado y Fernández-Bajón, 2016). Por ende, las universidades deben revisar de manera periódica las ofertas académicas afines a esta área para adaptarlas a las necesidades del momento.

De acuerdo con la Cámara de Tecnologías de Información y Comunicación, el sector de las Tecnologías Digitales ocupa una posición privilegiada en Costa Rica (CAMTIC PROCOMER, 2015), por lo que se deberían atender los requerimientos de las empresas que generan fuentes de empleo y colaboran con la economía.

Estudios nacionales recientes muestran algunos aspectos importantes a considerar referentes a las personas profesionales y los conocimientos y características que se requieren, como por ejemplo, habilidades blandas, un nivel superior de inglés y especialización en diferentes temas; también la necesidad de nuevas carreras que abarquen sectores del mercado que aún no han sido cubiertos (CAMTIC - PROCOMER, 2015; Programa Estado de la Nación, 2014; PROSIC, 2019). 
Sin embargo, a nivel país, no se han detectado estudios recientes que evidencien que las necesidades de educación formal en el área de las TIC están siendo cubiertas en los diferentes planes de estudio de las universidades públicas, esto, acorde con los cambios tecnológicos. Además, existe poca información sobre el sector TIC (PROSIC, 2018) y su regionalización.

Para responder con certeza a la pregunta ¿las carreras que ofrecen las universidades públicas están actualizadas según los avances tecnológicos? se inscribió en el Centro de Investigaciones sobre Diversidad Cultural y Estudios Regionales (CIDICER) de la Universidad de Costa Rica, un estudio cuyo objetivo principal se centra en investigar las necesidades en el área de las TIC en el país, con el fin de elaborar un diagnóstico que sirva como insumo para los procesos de actualización de las opciones académicas existentes y la creación de nuevas carreras.

\section{Referente teórico}

\subsection{Tecnologías de Información y Comunicación}

La tecnología evoluciona a cada momento, para atender las distintas necesidades del ser humano, automatizar procesos y, en general, facilitar la ejecución de tareas. De acuerdo con Gutiérrez (2016), las TIC son elementos de hardware, software y sus dispositivos periféricos, utilizados en la generación, transformación, almacenamiento y transmisión de la información en sus distintos formatos. Estas se encuentran inmersas en muchas de las actividades que desarrollamos hoy día, desde el ámbito económico y empresarial hasta el social y ambiental.

La adecuada absorción de las TIC es lo que marca la diferencia; ArévaloAscanio, Bayona-Trillos y Rico-Bautista (2015) insisten en el concepto de capacidad tecnológica del individuo, que se relaciona con los conocimientos y habilidades que se tienen para utilizar, mejorar y adaptar las nuevas tecnologías.

\subsection{Tendencias y tecnologías emergentes}

Las TIC han producido una revolución digital en todos los aspectos de las sociedades modernas, creando el impulso principal para lo que se ha llamado la cuarta revolución industrial, en donde las TIC son ubicuas y altamente disruptivas (PROSIC, 2018).

Las ciencias y tecnologías convergentes son aquellas que anteriormente se estudiaban por separado, pues no tenían relación directa aparente, pero que se han ido fusionando y se 
están utilizando para resolver problemas complejos que anteriormente parecían imposibles. En el ámbito nacional, el Ministerio de Ciencia, Tecnología y Telecomunicaciones (MICITT) las resume en cuatro grandes áreas (MICITT, 2015):

1. Info (ubicuidad de la información)

2. Bio (control sobre la biología)

3. Nano (economía en átomos)

4. Cogno (comprensión progresiva de la mente humana)

En el ámbito global, Gartner (2016) señala diez tendencias que seguirán hasta el año 2022:

- Inteligencia Artificial (automatización del comportamiento inteligente)

- Aplicaciones inteligentes y analíticas (procesamiento y análisis de grandes volúmenes de datos para la toma de decisiones)

- Internet de las cosas (interconexión digital de objetos cotidianos con internet, que recopilan y transmiten datos)

- Gemelos digitales (representaciones digitales de un producto o proceso que se utiliza para optimizar su diseño y uso)

- Nube en la frontera (máximo aprovechamiento de las capacidades de los dispositivos conectados a la nube)

- Plataformas conversacionales (sistemas informáticos con los que es posible mantener una conversación)

- Experiencia inmersiva (realidad virtual y realidad aumentada)

- Cadena de Bloques (tecnología que facilita el registro y confirmación de diferentes tipos de transacciones de manera confiable)

- Modelo impulsado por eventos (mezcla de entornos físicos y virtuales para mejorar la experiencia digital del usuario)

- Confianza y riesgo adaptativo continuo (seguridad fluida y adaptable)

\subsection{Sector Tecnológico de Costa Rica}

La globalización y los tratados de libre comercio propician toda una reacción de cambios en cadena. La globalización de la industria de los distintos servicios de información, incluyendo la informática, es un claro ejemplo de esto. Es posible trabajar desde Costa Rica para una empresa estadounidense que atiende a clientes en Europa. Además, la influencia 
y hegemonía de las empresas over the top (OTTs) tales como WhatsApp y Skype, que ofrecen servicios de telecomunicaciones utilizando la infraestructura de las compañías telefónicas tradicionales, son las que fuerzan las transformaciones del tipo de servicios.

Pasando del ámbito global al local, es necesario delimitar el concepto de tecnología de una manera que concuerde con lo que se desarrolla en el país. Es por eso que la Cámara Costarricense de Tecnologías de Información y Comunicación (CAMTIC) y la Promotora de Comercio Exterior de Costa Rica (PROCOMER) se encargaron de crear una clasificación de nueve subsectores de las tecnologías digitales en Costa Rica (CAMTIC - PROCOMER, 2015).

1. Desarrolladores de Software

2. Multimedia Digital

3. E-commerce

4. E-learning

5. Tecnología de la Información

6. Telecomunicaciones y Redes

7. Comercialización de Tecnologías

8. Servicios Habilitados por las Tecnologías Digitales

9. Manufactura de Componentes Digitales

Todos ellos en auge año con año, producen un impacto ya visible a nivel país. La exportación de servicios en TIC son el tercer servicio en importancia en Costa Rica, según datos de PROCOMER (2017). Esto debido al fortalecimiento y crecimiento del sector en actividades como centros de soporte técnico, centros de servicios empresariales, desarrollo de software, animación digital e ingeniería. Incluso, durante la crisis 2008-2009, las exportaciones de servicios empresariales y del sector de telecomunicaciones, informática e información tuvieron un crecimiento alto (Programa Estado de la Nación, 2017).

Además, las opciones académicas relacionadas a la ingeniería de software encabezan la lista de carreras con más opciones laborales en el sector servicios en el país, según los resultados de un estudio de demanda de capital humano efectuado a más de cien empresas multinacionales (CAMTIC - PROCOMER, 2015). 


\subsection{La educación superior en el área de las TIC}

Las universidades públicas del país tienen en su norte la visión humanista (UCR, 2005), entendida como la formación de profesionales con amplitud de criterios y no solamente especialistas con una posición tecnocrática. De acuerdo con el Consejo Nacional de Rectores y la Oficina de Planificación de la Educación Superior "Las universidades estatales de Costa Rica optan por ser instituciones humanistas, donde la ética, la capacidad crítica, la creatividad y el trabajo se constituyen en los pilares de desarrollo de las ciencias, las técnicas y las artes." (2011, p. 64). En este sentido, las personas profesionales complementan sus conocimientos específicos con una conciencia social que se corresponde con la preocupación de la universidad por la formación integral de la población estudiantil.

Existe una relación entre la realización profesional en el área de estudios y la valoración de la importancia en la educación recibida, pues de acuerdo con un estudio de Sanchez-Gelabert y Navarro-Cendejas (2016), los resultados muestran que cuando la formación académica no tiene un impacto positivo para conseguir o acceder a puestos de trabajo que correspondan al nivel de formación recibida, las personas egresadas tienden a tener una baja percepción de la utilidad de esta.

Es debido a estas circunstancias que se hace indispensable una conexión entre la oferta y la demanda, entre las opciones académicas ofrecidas y el mercado, sin olvidar, entonces, la parte humanista. La gestión de la enseñanza superior supone sobrepasar los siguientes retos: las distancias físicas son cada vez menos importantes, las culturas e idiomas se van acercando, la calidad y las competencias deben poder homologarse, el uso de espacios físicos o virtuales, entre otros. En la enseñanza transfronteriza, las TIC facilitan los procesos de enseñanza y democratizan su acceso (Fainholc, 2010).

\subsection{Formación continua como herramienta de desarrollo profesional}

Las universidades tienen un papel preponderante y una gran responsabilidad en la facilitación de espacios de formación continua para su población egresada. Los conceptos adquiridos en las aulas, con el pasar de los años, necesitan ser reforzados y actualizados, pues en todas las áreas se generan nuevos conocimientos: se desarrollan procedimientos quirúrgicos menos invasivos, se crean nuevas leyes, suceden nuevos acontecimientos mundiales que pasan a formar parte de la historia, y con la tecnología no es la excepción, la capacidad de procesamiento aumenta y también la interacción con más dispositivos. 
La formación continua busca la actualización profesional para que las personas puedan aplicar los nuevos conocimientos y competencias en su ambiente laboral o personal. Tiene la finalidad de que sean más competitivos y se cuente con las herramientas necesarias para generar innovación (Álvarez y Romero, 2007). Es también que, considerado un proceso educativo que no tiene fin, desde la perspectiva de Medrano, Repetto, Blanco y otros (2001), este proceso une los intereses profesionales particulares con los comunes, facilitando un entorno para compartir experiencias.

Una persona profesional en el área de las TIC debe procurar que esta experiencia de constante aprendizaje sirva para validar que se contemplen las necesidades sociales, las exigencias del empleo y las condiciones de la persona a lo largo de sus experiencias profesionales (Valenciano Canet, 2014).

\section{Metodología}

Este estudio inició en el mes de agosto del año 2016 y finalizó a inicios del año 2019, por lo que las actividades que se detallan a lo largo de la metodología se realizaron durante ese período.

\subsection{Enfoque}

El enfoque de este artículo es cualitativo, hace uso de recolección y análisis de datos mediante un proceso inductivo, que va de lo particular a lo general, con el fin de consolidar la teoría y establecer los patrones de comportamiento de la población de estudio (Hernandez Sampieri, Fernández Collado y Baptista Lucio, 2014). Basado en este marco metodológico, el propósito fue indagar sobre las necesidades actuales de educación superior universitaria en el área de las TIC, sustentado en análisis de los planes de estudio de las carreras que ofrecen opciones académicas de las universidades públicas del país, la experiencia de las empresas empleadoras en los contextos locales y una revisión documental de informes recientes a nivel nacional que, según Schenkel y Pérez (2019), a partir de eso la investigación cualitativa ayuda a comprender las realidades locales.

\subsection{Unidades de análisis}

\subsubsection{Participantes}

Para la selección de participantes se contempló específicamente el área de las TIC y se dividió en tres categorías: los estudios a nivel nacional, las empresas que contratan a las personas graduadas de carreras afines y las opciones académicas. 
Primeramente, se analizaron diferentes estudios realizados en el país en los últimos cinco años relativos a las TIC, con el propósito de tener una visión general de la realidad actual de la sociedad costarricense: Programa Hacia la Sociedad y el Conocimiento (PROSIC, 2017, 2018), Estado de la Ciencia, la Tecnología y la Innovación (Programa Estado de la Nación, 2014) y el Mapeo Sectorial de las Tecnologías Digitales 2014 (CAMTIC - PROCOMER, 2015).

Referente a las empresas, se elaboró una base de datos de empresas empleadoras de personas graduadas de carreras del área de las TIC, para ello se hizo uso primeramente de la información de las empresas que forman parte de la Cámara de Tecnologías de Información (CAMTIC, 2018). Además, se solicitó el apoyo a la carrera del Bachillerato en Informática Empresarial en las diferentes sedes y recintos de la Universidad de Costa Rica, las cuales le pidieron a las personas graduadas de las carreras que participaran aportando información sobre sus lugares de trabajo, a fin de obtener un espectro de empresas a lo largo de todas las regiones del país. Esta base de datos incluyó información de alrededor de doscientas diferentes instituciones y empresas a nivel nacional, la mayoría de ellas dedicadas al área tecnológica.

En primera instancia, se solicitó a todas las empresas de las que se tenía información que participaran del estudio contestando un cuestionario, sin embargo, solo el diez por ciento de ellas lo respondió. Se decidió no realizar una muestra, que según Piña León, D’Espaux Salgado y De Rojas Gómez (2012) se refiere a una parte o subconjunto de la totalidad de la población a estudiar, pues la finalidad era obtener la mayor información posible de su parte.

En total se obtuvo la participación de veintiséis diferentes personas representantes de empresas, quienes accedieron a responder un cuestionario de manera confidencial, enfocado en conocer las necesidades de formación que se reflejan en los requerimientos actuales de la población graduada de carreras relacionadas con las TIC.

Por último, en cuanto a las opciones académicas se decidió incluir todas aquellas carreras relacionadas con TIC, que se imparten en las universidades públicas del país, abarcando así la población de la totalidad de las regiones en las que se ofrecen.

\subsubsection{Unidades de estudio}

La investigación se centró en las opciones académicas universitarias, específicamente de las carreras afines al área de las TIC de las universidades públicas del país, dado que la finalidad de los resultados del diagnóstico es que puedan ser utilizados para aportarle a las 
carreras insumos para su mejora continua. Se contemplaron todos los planes de estudio de las diferentes opciones a nivel de Bachillerato, Licenciatura y Maestría enfocadas en el área de las TIC de todas las universidades públicas del país: Instituto Tecnológico de Costa Rica (TEC), Universidad de Costa Rica (UCR), Universidad Nacional (UNA), Universidad Estatal a Distancia (UNED) y Universidad Técnica Nacional (UTN). Dichos planes se especifican en el apartado de Resultados de este artículo. Cabe mencionar que se revisó el perfil de egreso, la malla curricular, duración de los cursos y las temáticas abarcadas.

Es importante aclarar que el estudio no se refiere a personas, sino a carreras universitarias que puedan ser sujetos de mejora según las necesidades actuales del país, sin embargo, tiene un impacto directo sobre ellas, pues el fin es que estas generen profesionales de calidad acordes con dichas necesidades.

\subsection{Técnicas de recolección}

Para la recolección de los datos se hizo uso de los medios tecnológicos para obtener los insumos necesarios vía Internet, correo electrónico, redes sociales, mensajería electrónica y entrevistas en línea, ya que de acuerdo con Orellana López y Sánchez Gómez:

las TIC brindan al investigador la posibilidad de utilizar el audio, el vídeo, la imagen, los datos en textos escritos o hablados, todos recolectados mediante la mensajería del correo electrónico, de los foros de discusión, la observación en línea, el monitoreo o registros de todas las acciones generadas por los sujetos mediante un equipo informático (2006, p. 206)

Se utilizó la tecnología para asistir la investigación cualitativa (Cisneros Puebla, 2003) para colaborar y, en cierta medida, facilitar el trabajo de las personas investigadoras y el manejo adecuado de la información recopilada. En primera instancia, se realizó una búsqueda electrónica en los sitios web oficiales de las diferentes universidades públicas, a fin de conocer cuáles de sus opciones académicas comprenden las TIC. Seguidamente, se extrajeron los planes de estudio de cada una de ellas en formato digital para su análisis.

Los estudios nacionales elaborados por distintas entidades también fueron buscados por medio del Internet, en las páginas oficiales de cada una. Todos estos son de dominio público.

En el caso de la recolección de datos de las empresas, su información y el contacto de las personas representantes, se usaron diferentes medios para obtenerlos y crear una base 
de datos de los contactos. Se inició contactando a la Cámara de Tecnologías de Información (CAMTIC) para solicitar un listado de las empresas que la conforman y de este acercamiento con la institución se obtuvo un enlace de dominio público en su página web, por lo que de ahí se obtuvieron los nombres de contacto de empresas posicionadas en Costa Rica y relacionadas con las TIC. Con este listado se indagó en Internet a cada una de las empresas en busca de un contacto telefónico. Posteriormente, hubo una comunicación telefónica con el propósito de explicarles el objetivo del proyecto y solicitar el nombre y el correo electrónico de una persona representante a quien se pudiera contactar para pedirle que colaborara respondiendo un cuestionario.

Adicional a esto, y con el propósito de aumentar el listado ya existente, para incluir otras empresas que no formaran parte de la Cámara de Tecnologías de Información y que además pudieran ser de las diferentes regiones del país, se le solicitó colaboración a las personas encargadas de coordinar la carrera de Bachillerato de Informática Empresarial de las distintas sedes y recintos de la Universidad de Costa Rica: Sede de Guanacaste, Sede del Pacífico, Sede del Caribe, Recinto de Paraíso, Recinto de Guápiles, Recinto de Turrialba y Sede del Sur, quienes compartieron la información de las empresas en donde las personas estudiantes próximos a concluir su plan de estudios realizan pasantía de la Práctica Empresarial Supervisada. Las personas coordinadoras de la carrera también solicitaron la participación a su población graduada, obteniendo que algunas y algunos accedieron a enviar la información de sus empresas empleadoras para que fueran contactados.

Es importante aclarar que, por limitaciones de tiempo y porque no se contempló como parte de las actividades del proyecto inscrito, no se solicitó la colaboración de las otras universidades públicas para aumentar este listado de empresas empleadoras, lo que podría ser una limitante. Sin embargo, los resultados de los cuestionarios que se les aplicaron a las personas representantes de las empresas que participaron del estudio reflejan que estas cuentan con población graduada de todas estas universidades y sus respuestas las abarcan.

Una vez creada la base de datos con la información obtenida por los diferentes canales, se procedió a elaborar una entrevista virtual, registrada en una herramienta de encuestas en línea, que se les envió vía correo electrónico a todos los contactos, esto entre los meses de septiembre y octubre del año 2018. En este tipo de entrevistas se logra obtener información textual, pero es similar a una entrevista cara a cara, en cuanto a fortalezas y debilidades. Además, se reducen los costos de desplazamiento de las personas investigadoras y se puede abarcar una población distante geográficamente en poco tiempo. 
La persona entrevistada puede tomarse el tiempo que necesite para formular sus respuestas, la información aportada se mantiene íntegra y al ser anónimas se reduce la inhibición y sienten más confianza para compartir la información solicitada, entre otras ventajas (Orellana López y Sánchez Gómez, 2006).

Las consultas a las personas representantes de las empresas se dividieron en tres secciones: información de la persona participante, información propia de la organización participante e información alusiva a tecnologías emergentes. En la primera sección se buscaba conocer el rol de quien completa la entrevista dentro de la empresa y su experiencia. La segunda sección tenía como objetivo ilustrar el contexto y campo de acción de la empresa. Con la última sección se pretendía conocer las TIC utilizadas, los conocimientos y capacidades de las personas empleadas profesionales graduadas del área de las TIC, habilidades y conocimientos requeridos como requisitos de contratación, tipos de formación académica, entre otras.

\subsection{Procesamiento de análisis}

El procesamiento y análisis de los datos recabados se realizó en tres etapas: categorización y tabulación, comparación e interpretación de los resultados.

La categorización se llevó a cabo de acuerdo con los tres tipos de fuentes de datos planteados en el proyecto: estudios a nivel nacional, entrevistas a personas empleadoras y planes de estudio de las todas las carreras del área de las TIC de las distintas universidades públicas consideradas en esta investigación y que fueron indicadas en el apartado anterior.

La información obtenida de cada uno de estos insumos fue separada y tabulada tomando en cuenta sus características. En el caso de los planes de estudio, se contemplaron todas las carreras de bachillerato, licenciatura y maestría que se ofrecen en las cinco universidades públicas del país, revisando perfil de egreso, la malla curricular, duración de los cursos y las temáticas abarcadas en ellos.

Los estudios, por su lado, fueron analizados y de ellos se obtuvo la información relacionada con las necesidades del mercado nacional en cuanto a las TIC y a la población graduada de las carreras afines. Se tomaron en cuenta informes de distintas instituciones como CAMTIC, PROCOMER y el Estado de la Nación, entre otras.

Por último, se separó la información brindada por las personas representantes de las empresas empleadoras de acuerdo con las preguntas realizadas. De esta manera, se 
cubrieron los ejes principales del estudio, permitiendo contemplar el panorama desde tres aristas distintas, pero que tienen en común el país y el área de las TIC.

Posteriormente, se realizó la comparación de la información de las tres fuentes, logrando detectar vínculos claves con las similitudes (Schettini y Cortazzo, 2015) y las diferencias de las percepciones que tienen las universidades públicas con respecto a los resultados de los estudios nacionales recientes y también con lo que requieren las empresas empleadoras de las personas graduadas.

Finalmente, se interpretan los resultados basados en la pregunta que ha guiado esta investigación ¿cuáles son las necesidades de educación formal en el área de las TIC que no están siendo cubiertas por las carreras existentes? De esta manera, se consigue determinar qué requerimientos no han sido abarcados, hasta el momento en que se realiza este estudio, en la educación superior y que pueden ser una opción de mejora para la oferta académica existente, o incluso para la creación de nuevas carreras que puedan ofrecerle al mercado laboral el perfil de la persona graduada que requiere.

\section{Resultados}

\subsection{Estudios a nivel nacional}

Se abarcaron estudios recientes a nivel nacional que abordan la temática de las TIC: Mapeo Sectorial de Tecnologías Digitales 2014 (CAMTIC - PROCOMER, 2015), Seguimiento de la condición laboral de las personas graduadas 2011-2013 de las universidades costarricenses, consultadas en el año 2016 (Gutiérrez Coto, Kikut Valverde, Corrales Bolívar y Picado Madrigal, 2018), Informe Hacia la Sociedad de la Información y el Conocimiento (PROSIC, 2017, 2018, 2019) y el Informe del Estado de la Ciencia, la Tecnología y la Educación (Programa Estado de la Nación, 2014).

De los estudios se logró extraer un listado de las necesidades identificadas relacionadas con las TIC y con las personas profesionales del área. Primeramente, se observa la necesidad de contar con más población graduada que pueda atender la alta demanda del país, tal como lo indica el Estado de la Ciencia, la Tecnología y la Educación "En la educación superior conviene fomentar las carreras relacionadas con la CTI, a través de incentivos para que las universidades aumenten la matrícula en disciplinas consideradas prioritarias y que tengan demanda en el mercado laboral." (Programa Estado de la Nación 2014, p. 62). 
Se refleja el requerimiento de que la academia suministre la cantidad de personas profesionales requeridas por las empresas, en el área de las TIC, que colaboren con el crecimiento del ecosistema digital y por ende con el crecimiento del país, de acuerdo con CAMTIC - PROCOMER "En el periodo 2014-2016, la carrera de Ingeniería de software y computación (73\%) representa la mayor parte de la demanda de profesionales con título universitario, conforme a las previsiones de las empresas consultadas. En menor medida están presentes otras áreas como diseño gráfico (8\%) y animación digital (6\%)" (CAMTIC PROCOMER, 2015, p. 102).

Además, se evidencia la necesidad de nuevas opciones académicas en campos que aún no han sido abordados en el país, donde se mezclen los saberes de las TIC con la salud, tal como se plasma en el Informe Hacia la Sociedad de la Información y el Conocimiento, recientemente publicado:

hace falta mucha formación profesional del personal que trabaja en el sistema de salud en las TIC. Además, existe la necesidad de un plan de actualización y formación continuada en las TIC en salud. La escasez de este tipo de profesional es una de las grandes limitaciones para el desarrollo de las TIC en salud en Costa Rica (PROSIC, 2019, p. 277)

Carreras como Informática Biomédica, Biocomputación Clínica e Informática Biomédica son algunas de las opciones que sugiere dicho informe que deben crearse en el país para ofrecer profesionales con conocimientos en ciencias básicas, ciencias médicas, matemáticas y las tecnologías, para disponer de los recursos de las TIC en beneficio de la salud y la población.

Las habilidades blandas también son requeridas en las personas profesionales de informática, entre ellas se mencionan el pensamiento analítico, habilidad comunicativa y asertiva, creatividad, servicio al cliente, orientación al detalle y habilidades técnicas, trabajo en equipo, capacidad crítica y analítica, resolución de problemas, liderazgo, deseos de aprender, curiosidad investigativa para mantenerse a la vanguardia, adaptabilidad, saber priorizar, manejo de tareas múltiples, ética, compromiso, honestidad e integridad, innovación y actitud emprendedora (CAMTIC - PROCOMER, 2015; Gutiérrez Coto et al., 2018; Programa Estado de la Nación, 2014).

Los estudios demuestran la necesidad de que las personas profesionales en esta área tengan dominio de un segundo idioma y resalta el inglés (CAMTIC - PROCOMER, 2015; 
Programa Estado de la Nación, 2014; PROSIC, 2017). Según lo indica el Informe del Programa Sociedad de la Información y el Conocimiento del año 2017 "La lengua es un obstáculo en la brecha digital, en particular se resalta la predominancia del inglés como vector de mundialización, y la brecha generada por no conocer este idioma" (PROSIC, 2017, p. 193).

Por último, los diferentes estudios consultados indican que se requiere mayor especialización en temáticas que ya se abordan en los planes de estudio de las carreras del área de las TIC, como lo son administración de proyectos, desarrollo de software interactivo, soporte aplicaciones tipo SAP y ERPs, computación en la nube, virtualización, ciberseguridad, pruebas de software, bases de datos y Big Data, diseño de hardware, manejo de infraestructuras de redes robustas y flexibles, redes inalámbricas, comunicaciones unificadas, Internet de las cosas (IOT), animación digital, entre otras.

Si bien, los estudios reflejan la realidad nacional actual, otros autores confirman las necesidades detectadas. Por ejemplo, Marrero y otros indican la necesidad de que la población estudiantil universitaria aprenda habilidades blandas que le permitan desempeñarse en el ambiente laboral (Marrero Sánchez, Mohamed Amar y Xifra Triadú, 2018). También, se destaca la necesidad de conocimientos tecnológicos claves como el Big Data, que ayuda a obtener información de grandes bases de datos facilitando su análisis y gestión, lo cual puede brindar ventajas competitivas a las empresas (Alegre, Ariño y Canela, 2017; Hernández-Pérez, 2016; Morato et al., 2016).

\subsection{Entrevista a las personas representantes de las empresas empleadoras}

Con el fin de conocer la opinión de las personas representantes de las empresas que emplean a las personas graduadas de carreras del área de las TIC, se elaboró un cuestionario para obtener la información, este les fue enviado vía correo electrónico a todos los contactos de alrededor de doscientas empresas del país que se incluyeron en la base de datos elaborada durante de esta investigación. Como resultado de este ejercicio se logró obtener veintiséis diferentes respuestas.

El objetivo del cuestionario era conocer sobre las tecnologías que están utilizando actualmente las empresas para el desarrollo de sus proyectos de software, el nivel de capacitación que tienen las personas profesionales que contratan en esas áreas y cuáles nuevas tecnologías quieren incorporar o están incorporando, todo esto abarcado en dieciocho preguntas. A continuación, se desglosan los resultados obtenidos. 
Se consultó a las personas entrevistadas por la posición que ocupan actualmente dentro de la empresa, la gran mayoría pertenece a posiciones de Gerencia $(46,15 \%)$ o Dirección (23,07\%). Esto es importante, pues en esas posiciones quienes participaron tienen gran conocimiento de la organización en general, del mercado, de los proyectos que se desarrollan en la actualidad y de las proyecciones a corto o mediano plazo de nuevas tecnologías para sus proyectos. En menor medida, ocupan otros tipos de posiciones como Ingeniería (11,53\%), Mercadeo (3,84\%), Consultor (3,84\%), Otros (11,53\%).

Con respecto a la cantidad de años que tienen de laborar, el $77 \%$ indicaron que tienen más de cinco años de trabajar para la misma empresa, lo cual demuestra que la mayoría de ellos tiene suficiente experiencia. Esto, aunado a lo indicado anteriormente sobre los puestos, hace que la percepción general del negocio y las necesidades de contratación sean mucho más amplias. Un 12\% indicaron que tenían de 3 a 5 años de trabajar para la misma empresa y un $11 \%$ tienen 2 años o menos.

En la misma línea, se les consultó si en el pasado habían asumido algún otro puesto dentro de la misma empresa, esta pregunta era opcional por lo que obtuvimos un total de 15 respuestas de las cuales la mayoría han estado en puestos de desarrollo, como ingenieros (en desarrollo, frontend, bases de datos), o de gerencia. El que las personas participantes tuvieran otras funciones o posiciones dentro de la misma empresa proporciona mayor experiencia y visión de la forma de trabajo dentro de la compañía y del negocio en general.

Otra de las preguntas realizadas fue por el rango de años de experiencia que tienen en el área de las TIC, el 73\% indicó que tiene más de 10 años de experiencia, mientras que un 15\% tiene de 6 a 10 años. Lo anterior muestra que casi 3/4 partes son profesionales con amplia experiencia en el área de TIC.

Según el informe de Estadísticas de Empresas de la Cámara de Comercio de Costa Rica (Cámara de Comercio de Costa Rica, 2015.), las empresas se pueden clasificar según la cantidad de trabajadores en: microempresas, entre 1 y 5 trabajadores; pequeñas empresas, entre 6 y 30 trabajadores; medianas empresas, entre 31 y 100 trabajadores y grandes empresas más de 100 trabajadores. En la muestra de las empresas que participaron respondiendo esta entrevista, el $58 \%$ se ubica en el rango de las pequeñas y medianas empresas (PYMES), un 27\% están entre las micro y pequeñas empresas, y el restante $15 \%$ son empresas grandes. Cabe mencionar que ninguna empresa se ubicó en el rango de 501 a 2500 colaboradores. Al ser la gran mayoría, pequeñas y medianas empresas, y 
conociendo que tienen más de 5 años de existir, se puede decir que son maduras y que cuentan con experiencia en el mercado y sus demandas actuales a nivel tecnológico.

Se consultó a las personas participantes sobre la universidad de procedencia de sus colaboradores graduados del área de las TIC, para esta pregunta el entrevistado podía elegir múltiples opciones y se le ofreció la lista de las cinco universidades públicas del país. De las veintiséis personas participantes, veintidós indicaron que tienen personal graduado de la UCR, por su parte, diecisiete indicaron que del TEC, nueve de la Universidad Nacional, seis de la UNED y tres de la UTN. Más de tres cuartas partes de las empresas han contratado personas profesionales de la UCR y más de la mitad del TEC, lo que podría indicar que la mayor parte de los resultados de esta encuesta evalúa el nivel de la población graduada de las carreras de informática que ofrecen estas dos universidades.

Los departamentos o unidades de investigación y desarrollo son importantes dentro de la empresa, pues reflejan que parte del presupuesto de esta se destina a incursionar en nuevas tecnologías, innovar, mejorar los sistemas existentes, crear patentes, entre otros. De las empresas participantes, el $62 \%$ indicó que no poseen un departamento de investigación y desarrollo. Esto es esperable en la mayoría de las empresas, pues son de tamaño pequeño o mediano, las cuales buscan generar capital y crecer, y este tipo de departamentos por lo general no genera ganancias a corto plazo, pues lo que se busca es ofrecer nuevas herramientas a las empresas para innovar.

De quienes participaron en representación de las empresas, indicaron que sí tienen departamento de Investigación y Desarrollo, el 60\% mencionó que lo conforman entre 1 y 5 colaboradores, seguido por un $20 \%$ que indicó que tienen de 11 a 30 . La mayoría de las empresas que tienen departamentos de investigación y de desarrollo, estos son pequeños, lo que va en concordancia con el tamaño de la empresa.

A las personas participantes se les ofreció una lista con nueve diferentes áreas que forman parte de las TIC para que seleccionaran en cuál o cuáles se centran principalmente las actividades de la empresa en la que laboran. La mayoría de las empresas se dedican al desarrollo de software (16 empresas), tecnologías de la información (13 empresas) y en menor medida a al comercio electrónico o e-Commerce (6 empresas).

Otro de los aspectos importantes a conocer son las principales regiones geográficas donde se ubican la clientela o personas usuarias de las empresas. En primer lugar, está Costa Rica (19 empresas), seguido por Latinoamérica y el Caribe (14 empresas) y, en tercer lugar, Estados Unidos y Canadá (10 empresas). En menor medida, hay clientes de Europa (5 
empresas) y solo una empresa indicó tener clientes en Asia. Además, ninguna tiene clientes en África ni en Oceanía. Basados en esto, se puede decir que la gran mayoría de los negocios de las empresas participantes se centran en países latinoamericanos y principalmente en nuestro país.

Con el propósito de conocer en cuáles industrias tecnológicas han desarrollado proyectos las empresas, se les ofreció a las personas participantes una lista con diecisiete opciones de selección múltiple en el cuestionario. Como se presenta en la Figura 1, se obtuvo que las cinco tecnologías en las que más se desarrollan proyectos son: aplicaciones en la web, sistemas para el sector público, sistemas de información (ERP, CRM, DSS), aplicaciones de la computación en la nube y telecomunicaciones. Los proyectos de Internet de las cosas y de servicios financiero-contables también tienen una participación importante.

Figura 1

Número de empresas encuestadas que han desarrollado proyectos según industria tecnológica, 2018.

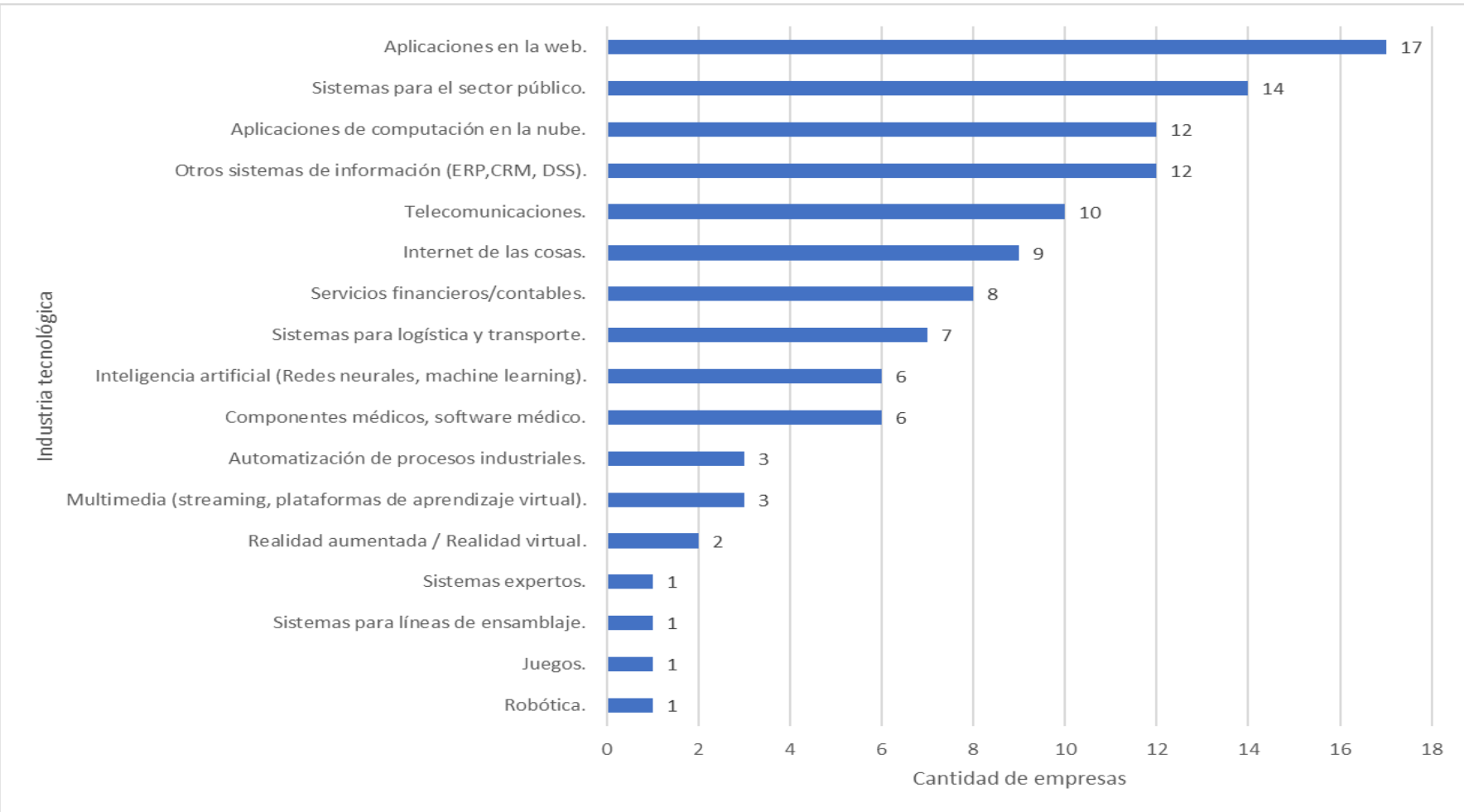

Fuente: Elaboración propia con resultados de encuesta realizada a empresas, 2018. 
Además, se solicitó seleccionar de una lista de dieciséis tecnologías emergentes, aquellas en las que ya se está desarrollando o si tiene interés en empezar a iniciar proyectos y a qué tiempo plazo, o bien, si no hay interés en ellas. De acuerdo con la Figura 2, las tecnologías en las cuales la mayor cantidad de empresas participantes están desarrollando proyectos en la actualidad son Internet de las cosas, Análisis de Datos, Smart Workspace, Edge Computing, Machine Learning y Data Mining.

\section{Figura 2}

Número de empresas encuestadas según la tecnología que utilizan para el desarrollo de proyectos TIC, 2018.

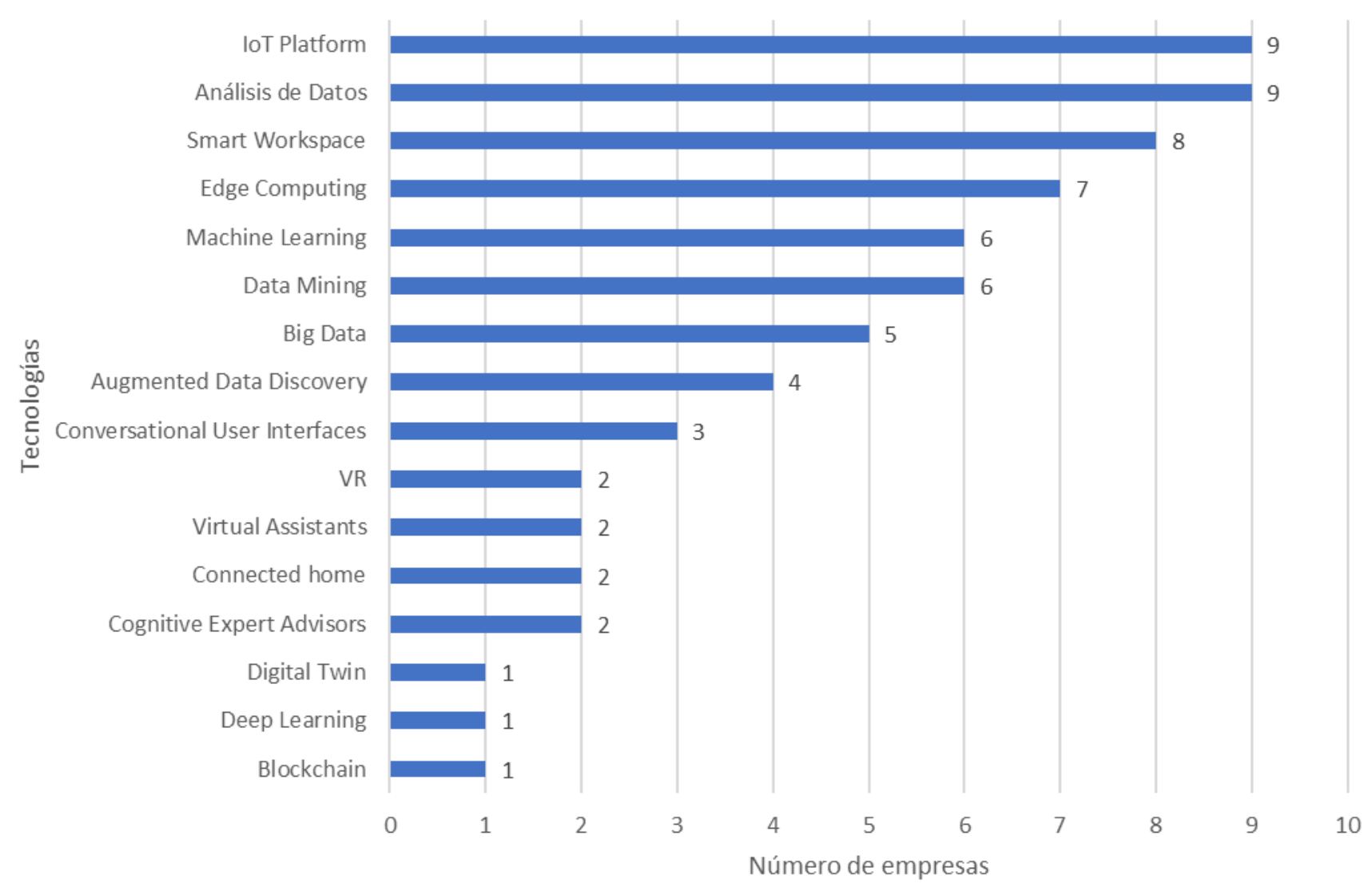

Fuente: Elaboración propia con resultados de encuesta realizada a empresas, 2018.

Las tecnologías en las que más interés tienen las empresas en los próximos años son Deep Learning, Big Data, Internet de las cosas, Machine Learning, Augmented Data Discovery y Virtual Assistants, como se muestra en la Figura 3. 
Figura 3

Número de empresas encuestadas que han desarrollado proyectos según industria tecnológica, 2018.

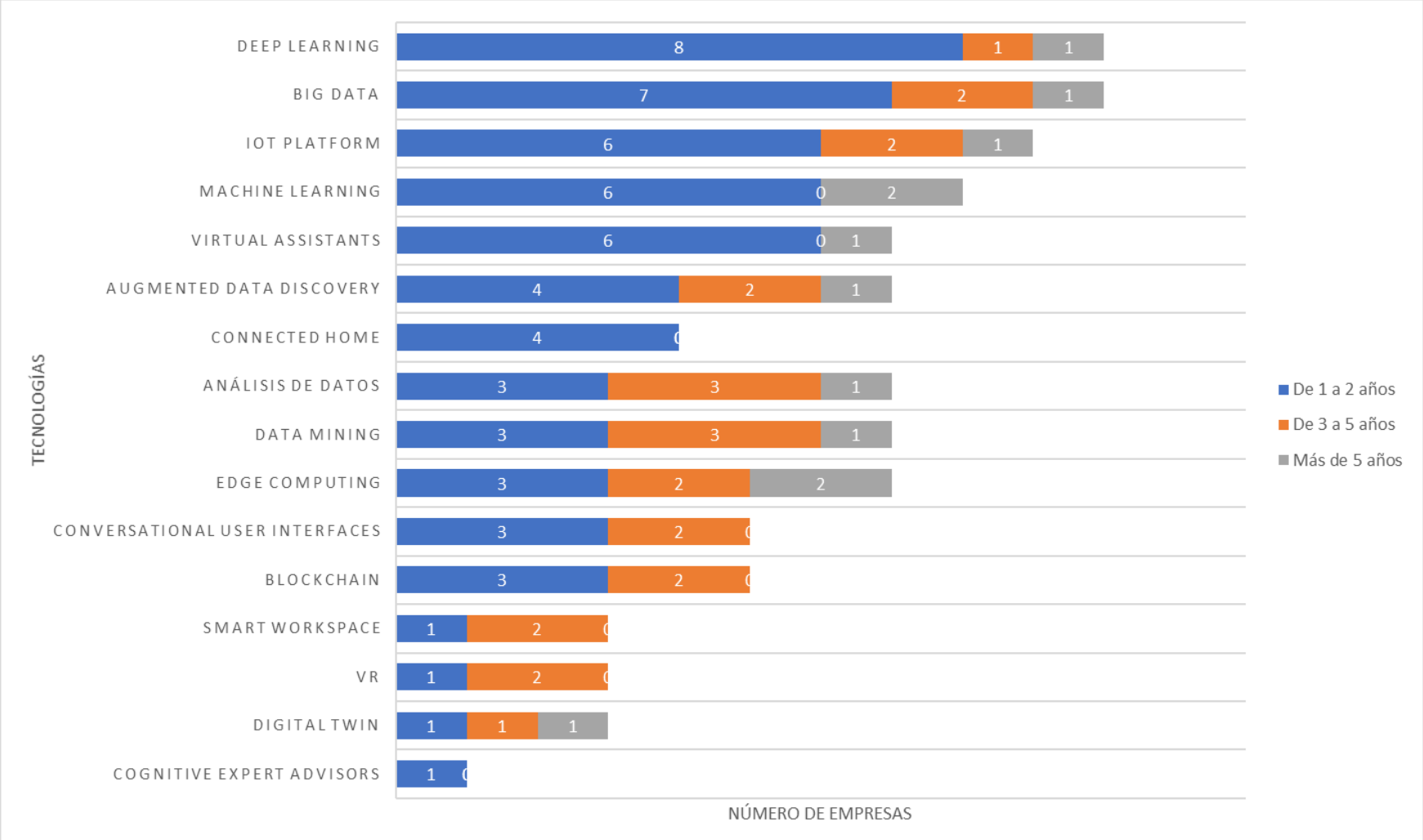

Fuente: Elaboración propia con resultados de encuesta realizada a empresas, 2018.

Resulta importante destacar que diez personas representantes de las empresas indicaron que tienen interés en desarrollar proyectos de Deep Learning, y de esas, ocho quieren hacerlo en un plazo de uno a dos años, mientras que actualmente solo una empresa está trabajando con proyectos en esa área.

Con respecto al uso de Big Data, seis indicaron que están desarrollando proyectos con esa tecnología en la actualidad, pero otras diez empresas están interesadas en incursionar en ella, siete de las cuales desean hacerlo en un plazo de uno a dos años.

En el caso de Internet de las cosas, nueve empresas actualmente desarrollan proyectos en este campo y otras nueve empresas están interesadas en dicha tecnología, seis de las cuales desean hacerlo en un plazo de uno a dos años.

Ocho de las personas participantes indicaron que sus empresas quieren desarrollar proyectos de Machine Learning, seis de estas quieren lograrlo en un plazo de uno a dos años. Otras seis indicaron que actualmente están desarrollando proyectos en esa área.

Por último, siete de las empresas tienen interés en desarrollar proyectos de Virtual Assistants, Augmented Data Discovery, Análisis de Datos, Data Mining y Edge Computing. 
De ellas, Análisis de Datos es la que más se desarrolla en la actualidad por las empresas, seguida por Edge Computing y Machine Learning.

Al consultar a las personas encuestadas sobre el nivel que capacitación que considera tiene la población profesional en el área de las TIC se obtuvo que hay una buena formación en áreas como: bases de datos programación web, móvil y de escritorio, así como en el área de redes. Sin embargo, ninguna de las empresas considera que existe capacitación suficiente en Blockchain, y de catorce empresas, diez consideran que la capacitación en Virtual Assistants es insuficiente, tal como lo muestra la Figura 4.

En tecnologías como Deep Learning, Big Data y el Internet de las cosas, que son de las que indicaron que tienen más interés en desarrollar en un futuro cercano, encontramos que la mayoría considera que la capacitación de los profesionales en esa área es insuficiente y en algunos casos básica.

Al haber respuestas de las empresas participantes que indican que en algunas tecnologías no se refleja una capacitación o esta es ineficiente o básica, se puede tomar como una opción de mejora en la formación de la población estudiantil en las carreras del área de las TIC.

\section{Figura 4}

Nivel de capacitación de la población profesional contratada por las empresas encuestadas, según el tipo de tecnología y cantidad de empresas que lo indicaron.

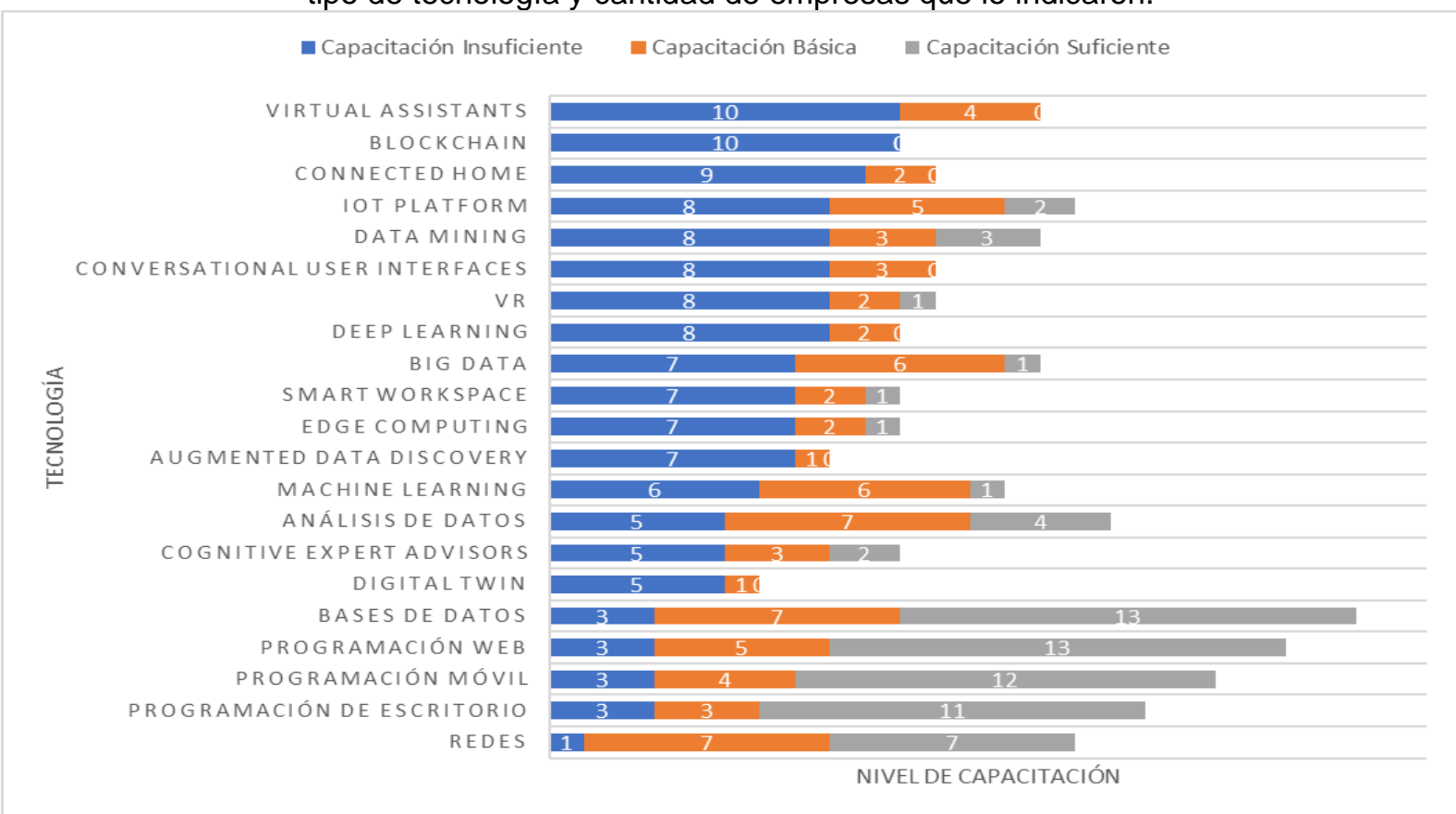

Fuente: Elaboración propia con resultados de encuesta realizada a empresas, 2018. 
Con respecto a los otros conocimientos o habilidades que se consideran importantes al momento de contratar a una persona profesional en el área de las TIC en la empresa mencionaron:

- SAP ERP

- Integración de datos

- Administración de procesos

- Habilidades blandas

- Ciberseguridad

- Análisis de procesos

- ITIL

- COBIT

- Linux

- Dirección de procesos
- Didáctica

- Inglés

- Animación

- Design Thinking

- Pensamiento crítico

- Comunicación

- Conocimientos avanzados en programación

- Conocimientos avanzados en bases de datos

- SCRUM

Al consultar por el tipo de formación académica universitaria que las empresas consideran necesaria para contratar a una persona profesional del área informática, la mayoría indicó que requieren en primer lugar un título de bachillerato y en segundo lugar que tengan cursos de certificación. En tercer y cuarto lugar se encuentran los técnicos y los diplomados. Incluso, los cursos libres están por encima de las opciones de maestría y doctorado como preferencia de formación para la población profesional. Queda claro que las empresas se enfocan más en el conocimiento técnico de ciertas áreas o tecnologías por encima de los grados superiores universitarios, como se aprecia en la Figura 5. 


\section{Figura 5}

Formación académica requerida para contratar a una persona profesional informática según las empresas encuestadas, 2018.

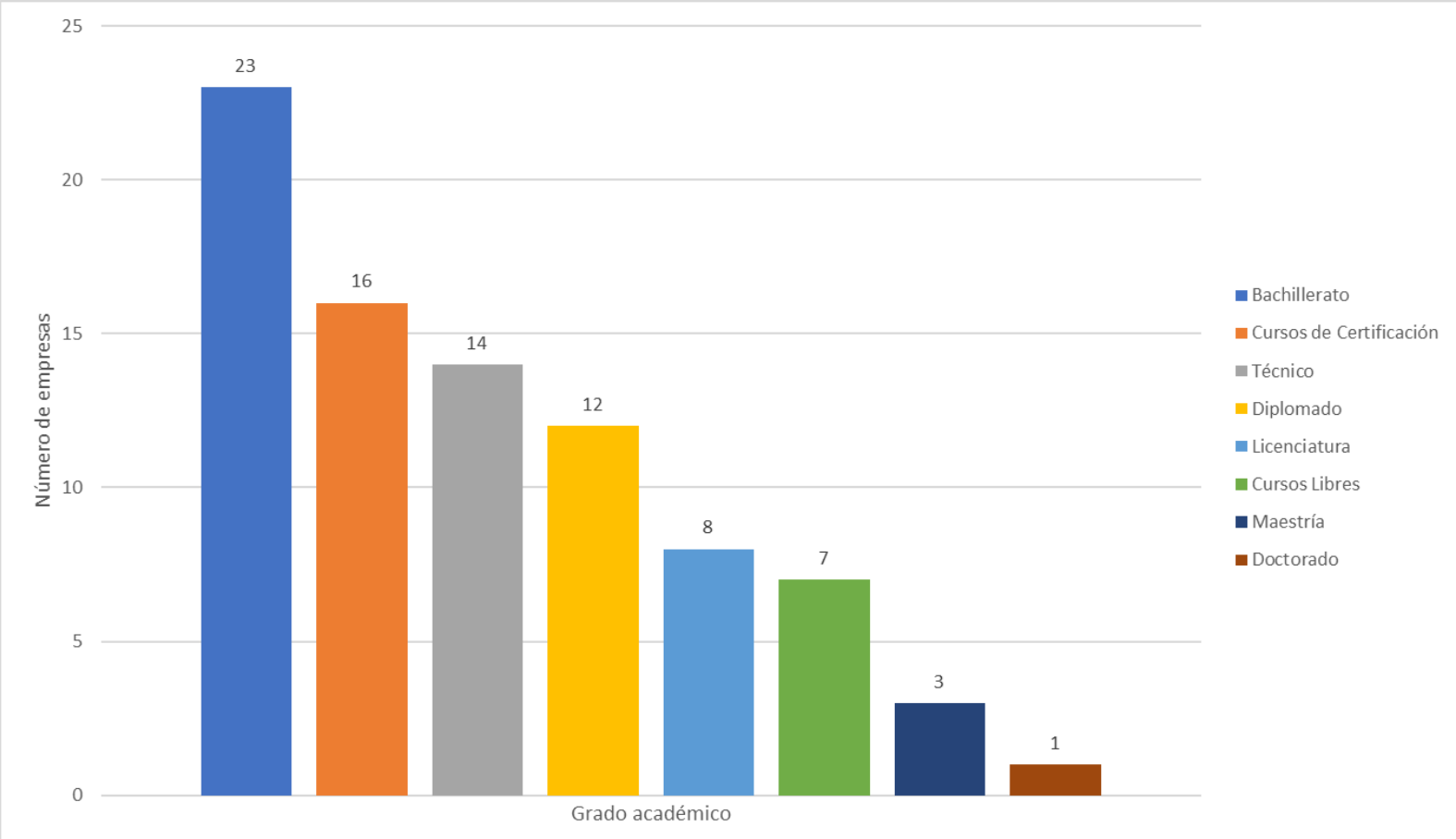

Fuente: Elaboración propia con resultados de encuesta realizada a empresas, 2018.

Por último, un $88 \%$ de las personas representantes de las empresas participantes indicaron que sí tienen interés en conocer los resultados de este estudio y un 96\% expresó anuencia a contribuir en futuros estudios o encuestas relacionadas con esta investigación.

\subsection{Planes de estudio de las carreras del área de las TIC en universidades públicas}

Se realizó la revisión documental de 25 opciones académicas entre diplomados y bachilleratos, licenciaturas y maestrías de las cinco universidades públicas del país: Universidad de Costa Rica (UCR), Instituto Tecnológico de Costa Rica (TEC), Universidad Nacional de Costa Rica (UNA), Universidad Estatal a Distancia (UNED) y Universidad Técnica Nacional (UTN), tomando en cuenta todas las carreras relacionadas con las TIC, tal como se aprecia en la Tabla 1. 
Tabla 1

Listado de opciones académicas en TIC por Universidad Pública Sedes en las que se imparten en Costa Rica, 2019.

Opción académica

Bachillerato en Informática Empresarial
Universidad

UCR

\section{Sedes en las que se imparte}

Sede del Atlántico (Recintos Turrialba, Paraíso y Guápiles), Sede del Caribe (Recintos Limón y Siquirres), Sede de Guanacaste (Recinto Liberia), Sede de Occidente (Recintos San Ramón y Grecia), Sede del Pacífico y Sede del Sur.

Sede del Pacífico (Recinto de Puntarenas)

\section{Tecnología Multimedia}

UCR

UCR

Bachillerato y Licenciatura en Ciencias de la Computación e Informática

Maestría en Auditoría en Tecnologías de la Información

Maestría en Bibliotecología y Estudios de la Información con énfasis en Tecnologías de la Información

Maestría Profesional en Computación e Informática

Maestría Profesional en Tecnologías de la Información y Comunicación para la Gestión Organizacional

Maestría Académica en Computación e Informática

Licenciatura en Administración de Tecnología de Información

Licenciatura en Ingeniería en Computadores

Bachillerato en Ingeniería en Computación

Maestría en Gerencia de Proyectos de Tecnologías de Información

Maestría en Computación con énfasis en Sistemas de Información
UCR

UCR

UCR

UCR

UCR

TEC

TEC

TEC

TEC

TEC
Sede Central Rodrigo Facio

Sede Central Rodrigo Facio

Sede Central Rodrigo Facio

Sede Central Rodrigo Facio

Virtual

Sede Central Rodrigo Facio

Sede Central Cartago

Sede Central Cartago

Sede Central Cartago; Sede Regional San Carlos; Centros Académicos: San José, Alajuela y Limón.

Sede Central Cartago

Centro Académico San José 
Bachillerato y Licenciatura Ingeniería en Sistemas de Información

Maestría en Gestión de la Innovación Tecnológica

Maestría en Tecnología e Innovación Educativa la Información

Bachillerato en Informática Educativa

Diplomado, Bachillerato y Licenciatura en Ingeniería Informática

Licenciatura en Ingeniería Informática y

Administración de Proyectos

Licenciatura en Ingeniería Informática y

Calidad del Software

Licenciatura en Ingeniería Informática y

Desarrollo de Aplicaciones Web

Maestría en Tecnología Educativa*

Diplomado, Bachillerato y Licenciatura en Ingeniería en Tecnologías de Información

Diplomado, Bachillerato y Licenciatura en Ingeniería del Software

UNA
Maestría en Administración de Tecnología de UNA

UNA

UNA

UNED

UNED

UNED

UNED

UNED

UNED

UTN

Fuente: Elaboración propia, con los planes de estudio de las diferentes opciones académicas relacionadas con TIC en las Universidades públicas de Costa Rica, 2019.

En cada caso se contactó a cada una de las instituciones educativas por medio de visita presencial o llamada telefónica para solicitar información de los planes de estudio. En todos los casos se indicó que estos se encontraban en las páginas web oficiales de la universidad, por lo que se consultaron y se hizo uso de los planes de estudio disponibles en ellas, se analizaron las mallas curriculares, el perfil de egreso, los cursos que las componen y la duración de los mismos, con el fin de encontrar puntos en común entre ellas y también diferencias que las destacan (UCR, 2019; TEC, 2019; UNA, 2019; UNED, 2019; UTN, 2019).

Se logró determinar que las carreras de grado comparten un bloque de cursos similares que abarcan las áreas de programación, bases de datos y lógica. La mayoría de ellas también tienen dentro de su plan de estudios, al menos un curso de redes, sistemas operativos, ingeniería de software, sistemas de información, arquitectura de software,

Los contenidos de este artículo están bajo una licencia Creative Commons
Campus Omar Dengo, Sede Brunca, Huetar Norte y Caribe.

Sede Central Campus Benjamín

Sede Central Campus Benjamín

Sede Central Campus Benjamín Núñez

A distancia

A distancia

A distancia

Guanacaste, Atenas, Sede

Pacífico

Bachillerato: Sedes Licenciatura:

Sedes Pacífico y Guanacaste

Sede Central y Sede San Carlos 
algoritmos y estructuras de datos. Además, en la mayoría de las opciones académicas se tienen también cursos relacionados con la informática y con la sociedad.

Destacan algunos cursos que no se repiten en ninguna otra carrera, por ejemplo: "Liderazgo y organización" del Bachillerato y Licenciatura en Ingeniería en Sistemas de Información de la UNA, "Gestión de proyectos" del Bachillerato en Informática Empresarial, el curso "Minería de datos" del Diplomado, Bachillerato y Licenciatura en Ingeniería del Software de la UTN o el de "Emprendedurismo de TI" en el Diplomado, Bachillerato y Licenciatura en Ingeniería en Tecnologías de Información de la misma universidad. Todos estos cursos necesarios hoy en día en los profesionales de las TIC y que dan un valor agregado a las personas graduadas de esas carreras.

Las opciones de posgrado muestran diferentes maestrías que, en su mayoría, están enfocadas en una temática en particular, entre ellas: auditoría informática, administración de proyectos o informática educativa por mencionar algunas. Como excepción están la maestría profesional y la académica en Computación e Informática que comparten un mismo listado de cursos optativos con diferentes opciones, en donde las personas estudiantes son quienes deciden el enfoque que le dan.

Sobresale la Maestría en Tecnología e Innovación Educativa y Maestría en Administración de Tecnología de la Información, ambas de la Universidad Nacional de Costa Rica que, dentro de sus planes de estudio, incluyen el curso "Liderazgo, destrezas efectivas y manejo de conflictos", que ofrece a su población estudiantil capacitación en habilidades blandas, que no se encuentran en ninguna otra opción de posgrado de las analizadas. Cabe mencionar que estas dos maestrías tienen planes de estudio renovados recientemente, en el 2018 y 2019 respectivamente.

\subsection{Necesidades de educación formal en el área de las Tecnologías de Información y Comunicación}

Luego de revisar los cursos que conforman las mallas curriculares de la oferta académica ofrecida por las universidades públicas, y de comparar estas con el listado de necesidades detectadas en los estudios revisados y en la información aportada por las empresas empleadoras participantes del estudio, se obtiene, a modo de resumen en la Tabla 2, las necesidades de educación formal en el área de las TIC. 
Tabla 2

Listado de necesidades de educación formal TIC por áreas en Costa Rica, 2019.

\begin{tabular}{|c|c|c|}
\hline $\begin{array}{c}\text { Nuevas opciones } \\
\text { académicas }\end{array}$ & $\begin{array}{ll}\text { - } & \text { Biocomputación Clínica } \\
\text { - } & \text { Informática Biomédica }\end{array}$ & $\begin{array}{l}\text { - Carreras de grado y posgrado } \\
\text { que contemplen las TIC y la } \\
\text { salud }\end{array}$ \\
\hline $\begin{array}{l}\text { Habilidades } \\
\text { blandas }\end{array}$ & $\begin{array}{ll}\text { - } & \text { Pensamiento analítico } \\
\text { - } & \text { Comunicación asertiva } \\
\text { - } & \text { Trabajo en equipo } \\
\text { - } & \text { Liderazgo } \\
\text { - } & \text { Deseos de aprender } \\
\text { - } & \text { Ética } \\
\text { - } & \text { Compromiso }\end{array}$ & $\begin{array}{ll}\text { - } & \text { Innovación } \\
\text { - } & \text { Servicio al cliente } \\
\text { - } & \text { Adaptabilidad } \\
\text { - } & \text { Manejo adecuado del tiempo } \\
\text { - } & \text { Trabajo bajo presión } \\
\text { - } & \text { Capacidad de análisis } \\
\text { - } & \text { Integridad }\end{array}$ \\
\hline $\begin{array}{l}\text { Temas de mayor } \\
\text { especialización }\end{array}$ & $\begin{array}{ll}\text { - } & \text { Administración de proyectos } \\
\text { - } & \text { Software interactivo } \\
\text { - } & \text { Computación en la nube } \\
\text { - } & \text { Virtualización } \\
\text { - } & \text { Ciberseguridad } \\
\text { - } & \text { Testeo } \\
\text { - } & \text { Realidad Virtual }\end{array}$ & $\begin{array}{ll}\text { - } & \text { Bases de datos, Big Data y } \\
& \text { Data Mining } \\
\text { - } & \text { Redes } \\
\text { - } & \text { Internet de las cosas } \\
\text { - } & \text { Animación digital } \\
\text { - } & \text { Machine Learning }\end{array}$ \\
\hline Idioma & - Dominio del inglés & - Segundo idioma \\
\hline
\end{tabular}

Fuente: Elaboración propia, con información de las mallas curriculares de las carreras en TIC de las universidades públicas, información aportada por la encuesta y estudios relacionados, 2019.

\section{Conclusiones}

Las conclusiones presentadas a continuación resultan del diagnóstico de las necesidades de educación formal en el área de las TIC en Costa Rica, y derivan del análisis documental de los estudios recientes a nivel nacional, de los planes de estudio de las carreras que componen la oferta académica afines en las universidades públicas, así como la opinión de las personas representantes de empresas empleadoras que participaron de la investigación.

El objetivo general se cumplió de manera satisfactoria, ya que se logró realizar un análisis de las necesidades en el área de las TIC en el país, ofreciéndole al lector un panorama actual sobre las habilidades, los conocimientos y las destrezas que la población estudiantil de las carreras afines a las TIC requiere para optar por puestos de trabajo una vez que se gradúen.

Los representantes de las empresas consultadas coinciden en que se requieren profesionales mejor capacitados en áreas como Blockchain, Virtual Assistants y Conected 
Home, además de una formación complementaria que permita desarrollar mejor las habilidades blandas.

Estos resultados podrían servir como insumo para procesos de actualización de carreras existentes en las otras universidades estatales y privadas del país, que busquen una mejora en sus planes de estudio para estar a la vanguardia, según las necesidades del mercado actual, así como también para la creación de nuevas opciones con énfasis específicos relacionados a las TIC, como Biocomputación Clínica o Informática Biomédica.

Es importante indicar que la carrera de Bachillerato en Informática Empresarial de la Universidad de Costa Rica, impartida actualmente en las diferentes sedes y recintos a lo largo de las distintas regiones, se encuentra en proceso de actualizar su perfil de salida y por ende su plan de estudios. Tal como se mencionó anteriormente, las personas investigadoras del presente proyecto son docentes de esta carrera, por lo que los resultados obtenidos serán compartidos con la Comisión de Docencia para que puedan valorar sus resultados en la mejora de los contenidos de los cursos existentes y la inclusión de temáticas que no se abordan actualmente, pero que son requeridas.

Además, las universidades pueden también valorar la posibilidad de crear programas de actualización continua para las personas graduadas de las carreras tecnológicas, tomando como base la información recolectada, ofreciéndoles herramientas de capacitación de calidad y de acuerdo con lo que requieren las empresas, que le brinden beneficios a nivel laboral y, además, que esta población siga manteniendo una relación con la academia.

Queda demostrado que se requiere crear nuevas opciones académicas de grado y posgrado en el área de las TIC, como Informática Biomédica, Biocomputación Clínica e Informática Biomédica, que no han sido cubiertas por las universidades públicas y que representan un nicho de oportunidad con el que se le podría ofrecer a la sociedad personas profesionales especializadas que vengan a llenar un vacío en áreas como la bioinformática, el análisis de datos masivos o la programación de aplicaciones en la nube.

Por último, se debe destacar que, aunque se realicen mejoras en las opciones académicas y se elaboren nuevas opciones de grado o posgrado, la necesidad de personas profesionales en el área de las TIC es alta. Según lo indicó la Cámara de Tecnologías de Información y Comunicación, en el país hay un faltante de 8.000 informáticos, y a pesar de que hay gran demanda de personas graduadas de colegio que desean estudiar carreras relacionadas con el área de las TIC, las universidades están limitadas en cuanto a la cantidad de cupos que ofrecen (CAMTIC, 2017). 
Por ser las TIC un área en constante avance, tal como lo menciona Sanz Lobo, Martínez Piñeiro y Permas Morado, "es comúnmente reconocido que las TIC forman parte del repertorio social actual de novedad, modernidad y prestigio, pero también de consumo y caducidad (objetos efímeros, rápidamente obsoletos, sucesión frenética de cambios...)" ( 2010, p. 37). Por lo que resulta importante que diagnósticos como este se realicen de manera frecuente, buscando siempre ofrecer las universidades información actualizada que pueda servirles para mantener una oferta académica consolidada que le brinde a la sociedad los profesionales de calidad que se requieren en las diferentes regiones.

\section{Agradecimientos}

Se agradece el apoyo que se tuvo por parte de todos los actores involucrados, CIDICER, las personas representantes de las empresas participantes de la investigación, universidades estatales, cuerpo docente y estudiantado. Gracias a todos y a todas se logró levantar una amplia lista de necesidades en el área de las TIC, que esperamos sean consideradas por la academia en un corto plazo.

\section{Referencias}

Alegre, Inés., Ariño, Miguel Ángel. y Canela, Miguel Ángel. (2017). Cómo sacar partido del análisis de datos big data. IEEM Revista de Negocios, 48(32), 46-51.

Arévalo-Ascanio, José Gregorio., Bayona-Trillos, Ramón Armando. y Rico-Bautista, Dewar Willmer. (2015). Responsabilidad social empresarial e innovación: Una mirada desde las tecnologías de la información y comunicación en organizaciones. Clío América, 9(18), 180-189. doi: $10.21676 / 23897848.1535$

Cámara de Comercio de Costa Rica. (2015). Estadísticas Económicas. Recuperado de http://camara-comercio.com/camara2/wpcontent/uploads/2015/11/17_docestadisticasempresas.pdf

Cámara Costarricense de Tecnologías de Información y Comunicación - Promotora de Comercio Exterior de Costa Rica. (2015). Mapeo sectorial de Tecnologías Digitales 2014. San José, Costa Rica: CAMTIC. Recuperado de https://www.camtic.org/estudios/mapeo-tic/

Cámara Costarricense de Tecnologías de Información y Comunicación. (6 de junio de 2017). Aumentan los profesionales en Informática y telecomunicaciones, pero no al ritmo en que lo demanda el sector. Recuperado de https://www.camtic.org/hagamosclic/aumentan-los-profesionales-en-informatica-y-telecomunicaciones-pero-no-al-ritmoen-que-lo-demanda-el-sector/ 
Cisneros Puebla, César A. (2003). Análisis cualitativo asistido for computadora. Sociologias, 5(9), 288-313.

Consejo Nacional de Rectores. Oficina de Planificación de la Educación Superior. (2011) Plan Nacional de la Educación Superior Universitaria Estatal 2011 - 2015. San José, Costa Rica. ISBN 978-9977-77-045-1

Fainholc, Beatriz. (2010). La formación científico-tecnológica digital en educación superior. RUSC: Revista de Universidad y Sociedad Del Conocimiento, 7(2), 1-11.

Gartner. (14 de octubre de 2016). Top 10 Strategic Technology Trends for 2017. Recuperado de https://www.gartner.com/en/documents/3471559/top-10-strategic-technology-trendsfor-2017

Gutiérrez Coto, Ilse., Kikut Valverde, Lorena., Corrales Bolívar, Karen. y Picado Madrigal, Cinthia. (2018). Seguimiento de la condición laboral de las personas graduadas 20112013 de las universidades costarricenses. San José, Costa Rica: CONARE. Recuperado de http://olap.conare.ac.cr/images/Proyectos/Seguimiento/2016/seguimiento_laboral_20112013.pdf

Gutiérrez González, Ángel. (2016). Tecnologías de la Información un enfoque interdisciplinario. México: Alfaomega.

Hernández-Pérez, Tony. (2016). En la era de la web de los datos: primero datos abiertos, después datos masivos. El Profesional de La Información, 25(4), 517-525. doi: 10.3145/epi.2016.jul.01.

Hernandez Sampieri, Roberto., Fernández Collado, Carlos. y Baptista Lucio, María del Pilar. (2014). Metodología de la Investigación (6a. ed.). México: McGraw-Hill.

Piña León, Luis., D’Espaux Salgado, Jorge. y De Rojas Gómez, Hugo. (2012). Técnicas de muestreo aplicadas a la actividad empresarial: la auditoría (II). Economía y Desarrollo, 145(1 y 2), 197-220.

Marrero Sánchez, Odalys., Mohamed Amar, Rachida. y Xifra Triadú, Jordy. (2018). Habilidades blandas: Necesarias para la formación integral del estudiante universitario. Revista Científica ECOCIENCIA, 5, 1-18.

Ministerio de Ciencia, Tecnología y Telecomunicaciones. (2015). Plan Nacional de Ciencia, Tecnología e Innovación (Vol. 01). San José, Costa Rica: MICITT. doi: 10.18259/ing.2016001

Morato, Jorge., Sánchez-Cuadrado, Sandra. y Fernández-Bajón, María Teresa. (2016). Tendencias en el perfil tecnológico del profesional de la información. El Profesional de La Información, 25(2), 168-178. doi: 10.3145/epi.2016.mar.03

Orellana López, Dania Ma. y Sánchez Gómez, Ma. Cruz. (2006). Técnicas de recolección de datos en entornos virtuales más usadas en la investigación cualitativa. Revista de Investigación Educativa, RIE, 24(1), 205-222. Recuperado de https://digitum.um.es/digitum/bitstream/10201/45434/1/Tecnicas de recoleccion de datos en entornos virtuales mas usadas en la investigacion cualitativa.pdf 
Promotora de Comercio Exterior de Costa Rica. (2017). Análisis trimestral sobre la evolución de las exportaciones de bienes y servicios de Costa Rica: Exportaciones de bienes al III trimestre de 2017 - Exportaciones de servicios al II trimestre de 2017. San José, Costa Rica: PROCOMER. Recuperado de https://www.procomer.com/uploads/downloads/efb018b94136733d738a22205b873db63 02a34d5.pdf

Programa Estado de la Nación. (2014). Informe Estado De La Ciencia, La Tecnología y la Innovación. San José, Costa Rica: PEN. Recuperado de https://estadonacion.or.cr/informes/

Programa Estado de la Nación. (2017). Programa Estado de la Nación. San José, Costa Rica: PEN. Recuperado de https://estadonacion.or.cr/informes/

Programa Sociedad de la Información y el Conocimiento. (2017). Programa Sociedad de la Información y el Conocimiento en Costa Rica: Informe 2017. San José, Costa Rica: UCR. Recuperado http://www.prosic.ucr.ac.cr/sites/default/files/recursos/informe_2017.pdf

Programa Sociedad de la Información y el Conocimiento. (2018). Hacia la Sociedad de la Información y el Conocimiento en Costa Rica: Informe 2018. San José, Costa Rica: UCR. $\quad$ Recuperado http://www.prosic.ucr.ac.cr/sites/default/files/recursos/final_informe_prosic_2018.pdf

Programa Sociedad de la Información y el Conocimiento. (2019). Programa Sociedad de la Información y el Conocimiento en Costa Rica: Informe 2019. San José, Costa Rica: UCR. Recuperado de http://www.prosic.ucr.ac.cr/informe-hacia-la-sociedad-de-lainformacion-y-el-conocimiento-2019

Sanchez-Gelabert, Alberth. y Navarro-Cendejas, José. (2016). La valoración que los graduados tienen sobre su formación universitaria desde su situación laboral actual. Revista Complutense de Educación, 27(2), 669-688. doi: 10.5209/rev_RCED.2016.v27.n2.47007

Sanz Lobo, María Dolores., Martínez Piñeiro, Esther. y Permas Morado, Eulogio. (2010). Innovación con TIC y cambio sostenible. Un proyecto de investigación continua. Profesorado, 14(1). Recuperado de https://www.redalyc.org/pdf/567/56714113018.pdf

Schenkel, Ericka. y Pérez, María Inés. (2019). Un abordaje teórico de la investigación cualitativa como enfoque metodológico. Acta Geográfica, 12(30), 227-233. doi: 10.5654/acta.v12i30.5201

Schettini, Patricia. y Cortazzo, Inés. (2015). Análisis de Datos Cualitativos en Investigación social. Procedimientos y herramientas para la interpretación de información cualitativa. Argentina: Editorial de la Universidad de la Plata.

Tecnológico de Costa Rica. (2019). Tecnológico de Costa Rica. Cartago, Costa Rica. Recuperado de https://www.tec.ac.cr 
Universidad de Costa Rica. (2005). Estatuto Orgánico de la Universidad de Costa Rica. San José, Costa Rica. Recuperado de http://www.cu.ucr.ac.cr/normativ/estatuto_organico.pdf

Universidad de Costa Rica. (2019). Universidad de Costa Rica. San José, Costa Rica. Recuperado de https://www.ucr.ac.cr/

Universidad Nacional de Costa Rica. (2019). Universidad Nacional de Costa Rica. Heredia, Costa Rica. Recuperado de https://www.una.ac.cr

Universidad Estatal a Distancia. (2019). Universidad Estatal a Distancia. San José, Costa Rica. Recuperado de https://www.uned.ac.cr

Universidad Técnica Nacional. (2019). Universidad Técnica Nacional. San José, Costa Rica. Recuperado de https://www.utn.ac.cr

Valenciano Canet, Grettel. (2014). Las necesidades de formación continua de las personas graduadas de la carrera de Orientación de la Universidad de Costa Rica y el apoyo que puede brindar la gestión educativa. Gestión de la Educación, 4(2). doi: 10.15517/rge.v4i2.15143

\section{Anexos}

La entrevista realizada a las personas representantes de las empresas empleadoras puede ser observada en el siguiente link: http://shorturl.at/fpuyS 
Revista indizada en

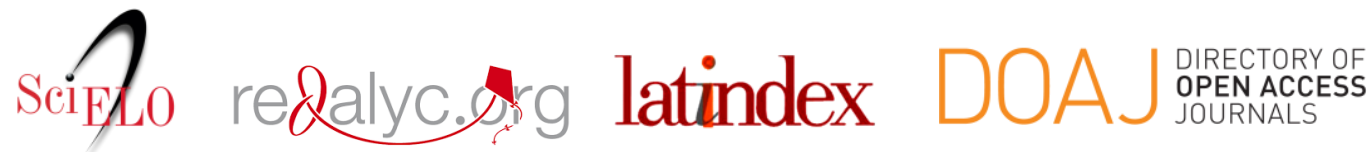

Distribuida en las bases de datos:

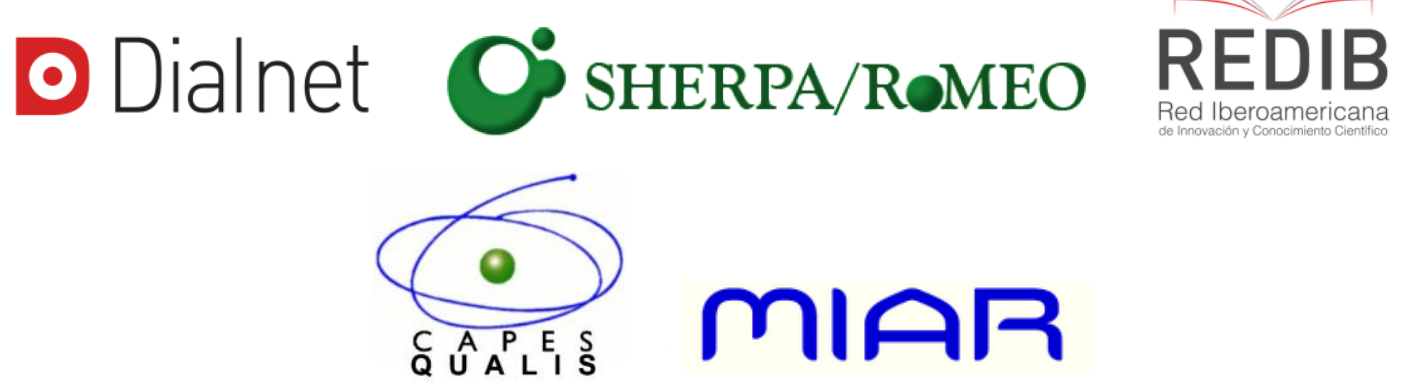

